\title{
P2X3 Receptor Ligands: Structural Features and Potential Therapeutic Applications
}

\author{
Andrea Spinaci, Michela Buccioni, Diego Dal Ben, Gabriella Marucci, Rosaria Volpini * and \\ Catia Lambertucci
}

Medicinal Chemistry Unit, School of Pharmacy, University of Camerino, Camerino, Italy

Keywords: $\mathrm{P} 2 \mathrm{X} 3$ receptor, $\mathrm{P} 2 \mathrm{X} 3-\mathrm{P} 2 \mathrm{X} 2 / 3$ receptor ligands, $\mathrm{P} 2 \mathrm{X} 3$ receptor agonists, $\mathrm{P} 2 \mathrm{X} 3$ receptor antagonists, drug discovery, Chronic cough, pain

\section{INTRODUCTION}

The ionotropic $\mathrm{P} 2 \mathrm{X} 3$ receptor $(\mathrm{P} 2 \mathrm{X} 3 \mathrm{R})$ subtype is one of the seven mammalian $\mathrm{P} 2 \mathrm{X}_{1-7}$ receptor belonging to the $\mathrm{P} 2$ purinergic receptor family together with the metabotropic $\mathrm{P}_{2} \mathrm{Y}_{1-2,4-6,11-14}$ ones (Fredholm et al., 2011). As the other P2X ion channels, it is a trimeric cell surface receptor permeable to $\mathrm{Na}^{+}, \mathrm{K}^{+}$, and $\mathrm{Ca}^{2+}$ cations and it is activated by the natural ligand adenosine- $5^{\prime}$-triphosphate (ATP, 1, Figure 1). Each subunit is constituted by two trans-membrane domains connected by a large glycosylated extracellular loop, which contains many disulfide bonds and the ATP binding site (Browne et al., 2010). P2X3Rs are assembled as homotrimers, constituted by three subunits of P2X3Rs, or heterotrimers, constituted by two P2X3Rs and one P2X2R subunits (P2X2/3Rs) (Lewis et al., 1995). A difference between the two forms is represented by their fast or slow desensitization after prolonged exposure to agonists; hence P2X3Rs undergo rapid inactivation/desensitization during exposure to ATP or to the selective agonist $\alpha, \beta$-methyleneATP ( $\alpha, \beta$-meATP, 2, Figure 1), which is accelerated by increasing the agonist dose, while P2X2/3Rs shows either mixed (twocomponent) or slow-type desensitization (Giniatullin and Nistri, 2013).

\section{P2X3 RECEPTORS AS TARGET FOR POTENTIAL DRUGS}

Both the homotrimeric P2X3 and heteromeric P2X2/3Rs are expressed on terminals of primary afferent sensory neurons of dorsal root ganglia (DRG), spinal cord and brain and their activation by ATP mediates pain sensation, hyperalgesia and allodynia in rodents (Jarvis, 2003). Notably, unlike rodents, the P2X3 subtype is the predominant ATP receptor subtype in human DRG sensory neurons (Serrano et al., 2012). On the other hand, the expression of the P2X2/3 heteromers is higher in afferents of viscera with respect to the somatic innervation, leading to potential different therapeutic approaches to treat visceral or somatic pain (with a P2X3 selective or a dual P2X3P2X2/3 antagonist) (Ford, 2012). The injection of ATP or $\alpha, \beta$-meATP into rodent skin induced nociceptive behavior (Kennedy, 2005; De Logu and Geppetti, 2019), whereas the use of the P2X3R antagonist $2^{\prime}, 3^{\prime}$-O-(2,4,6-trinitrophenyl)adenosine- $5^{\prime}$-triphosphate (TNP-ATP, 3, Figure 1) mediated antinociceptive effects (Honore et al., 2002). Hence, these receptors represent attractive targets to treat pain related disorders (Burnstock, 2018). P2X3 and P2X2/3Rs are functionally expressed also into the lamina propria, urothelium and detrusor smooth muscle of urinary bladder (Ford and Cockayne, 2011). It has been demonstrated that ATP or $\alpha, \beta$-meATP dose dependently stimulated bladder overactivity in conscious rats and this effect was antagonized by TNP-ATP. Hence, P2X3 and P2X2/3R antagonists are recognized as potential drugs to treat urological dysfunction, such as overactive bladder (Ford et al., 2006; Andersson, 2016). It is worthwhile to 


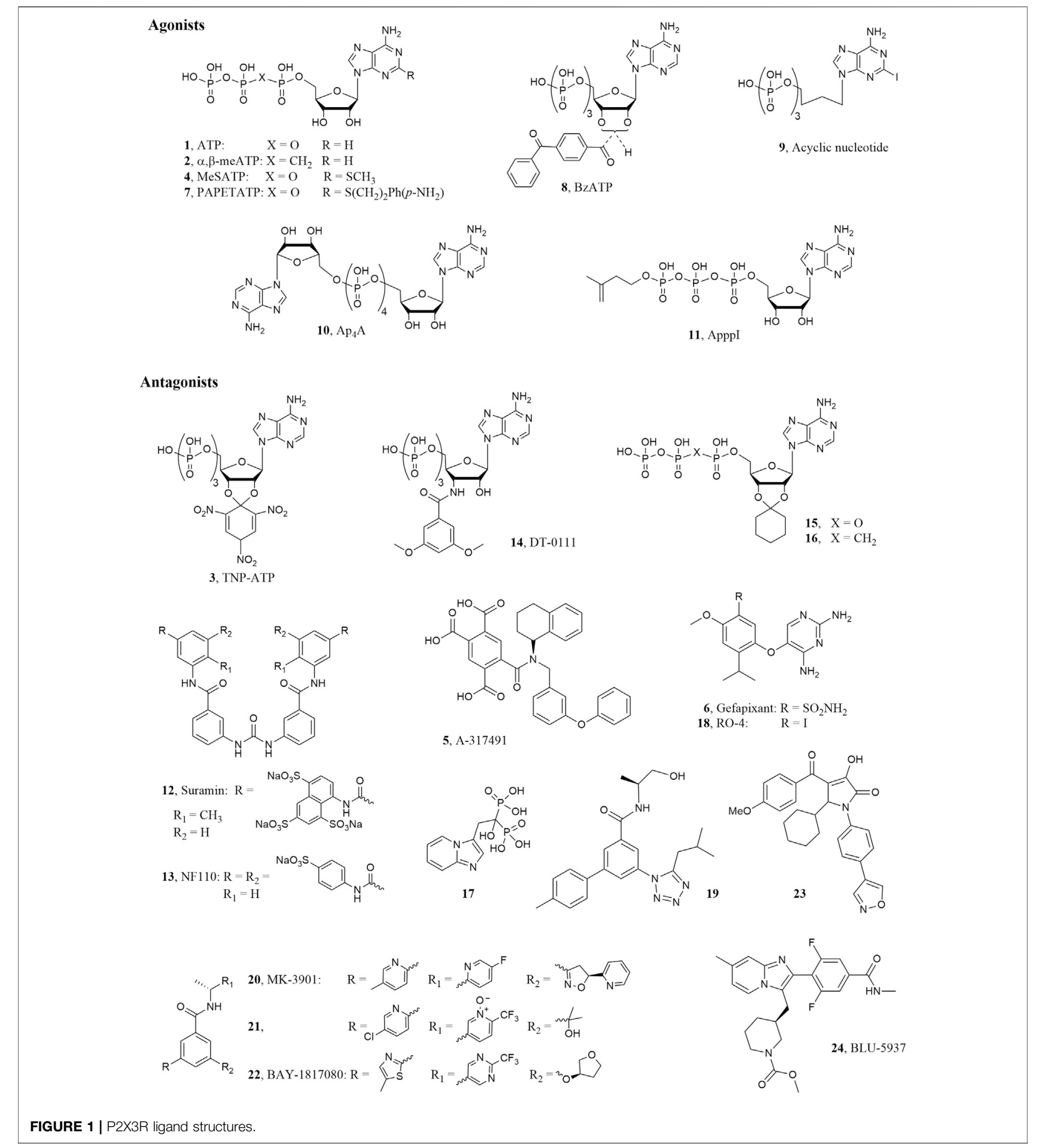

note that extracellular ATP, released in terminals of primary afferents in airway tissue or at the level of their central synapses, mediates protective reflex responses including cough, through interaction with P2X3 and P2X2/3Rs (Weigand et al., 2012).
This finding constitute the rationale for the use of these receptors antagonists in patient affected by chronic obstructive pulmonary disease (COPD) and chronic cough (Dicpinigaitis et al., 2020). 


\section{P2X3 RECEPTOR LIGANDS}

Most of the P2X3 agonists reported so far derived from modification of the ATP molecule, however, the search for molecules that activate these receptors is mainly of experimental interest. Due to the above mentioned therapeutic applications, in the last years many efforts have been directed through the discovery of potent and selective P2X3 and P2X2/3R antagonists derived both from high throughput screening of large compound libraries and rational design by molecular modeling studies, which were facilitated by the publication of the crystallographic structures of the P2X receptors. Beside in silico studies carried out with the development of theoretical 3D models of the P2X3 receptor (Grimes and Young, 2015). more recent works were based on experimental structures of the same protein. At present, several X-Ray structures of the P2X3 receptors are available in complex with agonists, i.e. ATP or 2-methylthioATP (2-MeSATP, 4), and ATP competitive (orthosteric) antagonists like TNP-ATP and 5-[[[(3-phenoxyphenyl)methyl][(1S)1,2,3,4-tetrahydro-1-naphthalenyl]amino] carbonyl]-1,2,4benzenetricarboxylic acid (A-317491, 5) or non-competitive (allosteric) antagonists like 5-(2,4-diamminopyrimidin-5yloxy)-4-isopropyl-2-methoxybenzenesulfonamide (AF-219, also called MK-6274 or gefapixant, 6, Figure 1) (Mansoor et al., 2016; Wang et al., 2018; Li et al., 2019).

\section{Agonists}

The natural ligand ATP $\left(\mathbf{1} ; \mathrm{EC}_{50}=0.5 \mu \mathrm{M}\right)$ (Jarvis and Khakh, 2009) is able to activate P2XRs with various degrees of affinity. Extracellular ATP is rapidly hydrolized by ecto-nucleotidas to form ADP, AMP, and then adenosine. Compared to ATP, ADP show weaker activity at P2X3Rs (Lewis et al., 1995; North and Surprenant, 2000). Chemical modification of ATP led to P2X3R agonists and also antagonists (Lambertucci et al., 2015). In particular, the introduction of substituents in the 2-position of ATP slightly increased the P2X3R affinity leading to the unselective agonist 2-MeSATP $\left(4 ; \mathrm{EC}_{50}=0.35 \mu \mathrm{M}\right.$ ) (Jarvis and Khakh, 2009), which binds also the other P2X subtypes, especially the P2X1Rs. On the contrary, the 2-substituted $p$-aminophenylethylthioATP (PAPET-ATP, $7 ; \quad \mathrm{EC}_{50}=$ $0.017 \mu \mathrm{M})$ resulted a potent and selective P2X3R agonist at rat receptor (Jacobson et al., 2006). Recent experiments of X-Ray crystallography were reported showing the binding mode of both ATP and 2-MeSATP at the human P2X3R, with information about the roles of the triphosphate chain and the $2^{\prime}$ - and $3^{\prime}$ hydroxyl groups in the ligand-target interaction (Mansoor et al., 2016; Li et al., 2019). Since the triphosphate chain of the ATP derivatives is easily and rapidly hydrolyzed by ectonucleotidases, stable analogues like $\alpha, \beta$-meATP, which activate P2X3Rs with $\mathrm{EC}_{50}$ in the sub $\mu \mathrm{M}$ range, were synthesized. Modification of the ATP sugar moiety resulted in the potent agonist benzoylATP (BzATP, 8; $\left.\mathrm{EC}_{50}=0.08 \mu \mathrm{M}\right)$ (Jarvis and Khakh, 2009), which is a mixture of the $2^{\prime}$ - and $3^{\prime}$-benzoyl esters, while its replacement with open chains gave acyclic nucleosides like 2-iodo-9butyladenine triphosphate $\left(9 ; \mathrm{EC}_{50}=0.08 \mu \mathrm{M}\right)$, which showed responses of $60 \%$ of the maximal effect elicited by $\alpha, \beta$-meATP in patch-clump assay, so behaving as partial agonist (Volpini et al., 2009).

Some naturally occurring diadenosine polyphosphates were found as unselective P2X agonists with micro- or submicromolar activities, with the tetra- and triphosphonate derivatives $\mathrm{Ap}_{4} \mathrm{~A}$ (10, Figure 1) and $A p_{3} A$ showing full and partial $r P 2 X 3 R$ agonist profile, respectively (both molecules being endowed with higher potency than ATP) (Wildman et al., 1999). Ap 4 A showed ability to readily desensitize hP2X3R at nanomolar concentrations (McDonald et al., 2002). In a recent work, chemically stable analogues of this molecule showed a similar ability to desensitize the human and rat P2X3Rs, with a weak partial agonist profile only at high concentrations (Viatchenko-Karpinski et al., 2016). Chemical modifications of the diadenosine polyphosphates led to development of the so-called nitrogen-containing bisphosphonates (NBPs), which are of clinical relevance in particular for bone diseases. Among these molecules, ApppI (11, Figure 1) showed to rapidly desensitize rat P2X3R at low nanomolar concentration (Ishchenko et al., 2017). The highpotency activation of the $\mathrm{P} 2 \mathrm{X} 3$ by these molecules, followed by rapid desensitization, makes them de facto inhibitors of this receptor, with a clinical potential as analgesics.

\section{Antagonists}

While most of the P2X3 agonists reported so far derived from modification of the ATP molecule, the antagonists belong to various chemical classes. The first orthosteric antagonists to be identified were the polysulphonated naphthylurea suramin, its derivatives and various histochemical dyes (Jacobson et al., 2002). Suramin $\left(12 ; \mathrm{IC}_{50}=3.0 \mu \mathrm{M}\right)$ (Lewis et al., 1995) and its derivatives behave as nonselective antagonists with $\mathrm{IC}_{50}$ in the micromolar range at P2X3Rs, with the exception of NF110 $\left(13, \mathrm{IC}_{50}=0.09 \mu \mathrm{M}\right)$, which displayed submicromolar activity at rat receptors. The ATP derivative $3^{\prime}$-deoxy-3'-(3,5dimethoxybenzamido)ATP (DT-0111, 14; $\mathrm{IC}_{50} 3.0 \mu \mathrm{M}$ ), bearing a dimethoxyphenyl amido group in place of the hydroxyl in $3^{\prime}$-position of the ribose, is recognized as a novel small water soluble molecule that behaves as a selective antagonist at $\mathrm{P} 2 \mathrm{X} 2 / 3 \mathrm{Rs}$. When administered as an aereosol in in vivo experiments, the compound inhibited bronchoconstriction and cough induced by ATP (Pelleg et al., 2019; Illes et al., 2020). The linking of the $2^{\prime}$ and $3^{\prime}$ ATP hydroxyl groups by a trinitrophenyl ring gave the compound TNP-ATP $\left(3 ; \mathrm{IC}_{50}=0.001 \mu \mathrm{M}\right)$, which resulted the most potent competitive antagonist with potency in the low nanomolar range (Lewis et al., 1998) (Lambertucci et al., 2015). Molecular modeling studies led to the design of derivatives in which the trinitrophenyl group of TNP-ATP was replaced by cycloalkyl or aromatic rings (Dal Ben et al., 2015; Dal Ben et al., 2017). Among them, the $2^{\prime}, 3^{\prime}-O$-cyclohexylideneATP (15, Figure 1) displayed an $\mathrm{IC}_{50}=0.083 \mu \mathrm{M}$. Modification of the triphosphate chain of this derivatives gave the $\alpha, \beta$-methylene stable analogue 16, which displayed an $\mathrm{IC}_{50}=17.5 \mu \mathrm{M}$ at human and $0.127 \mu \mathrm{M}$ at rat P2X3Rs, being selective vs. the other P2X subtypes (Dal Ben et al., 2019). The substitution of the cycloalkyl group of $\mathbf{1 5}$ with a small alkyl moiety maintained the antagonist 
activity, with the isopropylidene function being the smallest group allowed (Dal Ben et al., 2019).

The small non-nucleotide molecule A-317491 (5; $\mathrm{IC}_{50}=$ $0.02 \mu \mathrm{M})$ was the first identified potent and selective P2X3 and $\mathrm{P} 2 \mathrm{X} 2 / 3 \mathrm{R}$ competitive blocker. However, although in its structure are present three carboxylic groups, it is endowed with low water solubility and oral bioavailability (Jarvis et al., 2002). X-Ray crystallography experiments showed that A-317491 and TNPATP bind in the same cavity of ATP hence confirming the ATPcompetitive mechanism of action, with the tetracarboxylic acid moiety of A-317491 occupying the same position adopted by the triphosphate chain of TNP-ATP (Mansoor et al., 2016).

Few years later, medicinal chemistry efforst by AstellasPharma led to the development of imidazopyridine derivatives with activity on P2X2/3 receptors (Kakimoto et al., 2008). Among these compounds, minodronate (17, Figure 1) was approved to market in Japan for the treatment of osteoporosis given its ability to inhibit of farnesyl pyrophosphate synthase; its additional activity on $\mathrm{P} 2 \mathrm{X} 2 / 3$ resulted an advantage to reduce low back pain in patients (Ohishi and Matsuyama, 2018).

Later on, a number of diaminopyrimidine derivatives were reported by the company Roche as potent and selective negative allosteric modulators of P2X3 and P2X2/3Rs like the 5-(5-iodo-2isopropyl-4-methoxyphenoxy)pyrimidine-2,4-diamine (RO-4 or $\left.\mathrm{AF}-353,18 ; \mathrm{IC}_{50}=3.16 \mathrm{nM}\right)$ or gefapixant $\left(6 ; \mathrm{IC}_{50}\right.$ of 0.03 and $0.250 \mu \mathrm{M}$ at $\mathrm{P} 2 \mathrm{X} 3$ and $\mathrm{P} 2 \mathrm{X} 2 / 3 \mathrm{Rs}$, respectively), which are endowed with favorable pharmacokinetic profile (Carter et al., 2009; Gever et al., 2010; Ford and Undem, 2013). X-Ray crystallography experiments showed that gefapixant binds the $\mathrm{P} 2 \mathrm{X} 3$ at the interface between the receptor subunits, in a different binding pocket than ATP. Interestingly, the substituted benzyl moiety of this molecule occupies a sub cavity that is partially occupied also by the trinitrophenyl group of TNP-ATP (Mansoor et al., 2016; Wang et al., 2018). Replacement of the pyrimidine scaffold of these molecules with a purine led to derivatives that, although less potent, retained the ability to block the receptors (Lambertucci et al., 2013). Given the interesting therapeutic potential of molecules that block P2X3 and P2X2/3Rs, in recent years a number of antagonists endowed with a good pharmacokinetic profile and reasonable oral bioavailability have been discovered and reported in numerous patents by pharmaceutical companies (Marucci et al., 2019). Among the diaminopyrimidine derivatives, the most promising one reported from Roche and developed by Merck is the above cited gefapixant (6), which was studied in clinical trials for different pathologies like idiopathic bladder disorders, osteoarthritic joint pain, and pulmonary cystic fibrosis (Jarvis, 2021). In patients with refractory chronic cough, gefapixant dose dependently reduced awake cough frequencies, however it induced alteration of taste sensitivity (dysgeusia) and, in some cases, the completed loss of taste (ageusia). The loss of taste response seems to be due to its unselective block of P2X3 and P2X2/3Rs. Although these side effects, the medical need for patients suffering of this pathology, encouraged Merck to continue the development of gefapixant, which has recently completed two Phase III clinical trials (called COUGH-1 and COUGH-2; NCT03449134, 2020; NCT03449147, 2020) for refractory or unexplained chronic cough (Muccino et al., 2020). In these studies, gefapixant at the dose of $45 \mathrm{mg}$ twice daily induced a significant reduction in 24-h cough frequency with mild to moderate alteration of taste sensitivity (Merck News release, 2020).

Also arylamide derivatives were found as potent and selective P2X3R antagonists. In particular, the 5-(5-isobutyltetrazol-1-yl)$4^{\prime}$-methylbiphenyl-3-carboxylic acid ((S)-2-hydroxy-1methylethyl)amide $\left(19 ; \mathrm{pIC}_{50}=8.8\right)$, reported by Roche company, resulted the most active compound at P2X3Rs in in vitro functional experiment performed using FLIPR (Fluorometric Imaging Plate Reader) Assay (Dillon et al., 2015). Another potent and selective P2X3R antagonist belonging to the arylamide derivatives was found by Merk. This compound, named MK-3901 ( $N$-[1(R)-(5-fluoropyridin2-yl)ethyl]-3-(5-methylpyridin-2-yl)-5-[5(S)-(2-pyridyl)-4,5dihydroisoxazol-3-yl]benzamide, 20), was tested in a $\mathrm{Ca}^{2+}$ mobilization FLIPR assay (FLIPR IP $=21 \mathrm{nM})$ and showed an efficacy comparable to that of naproxen in a rat inflammatory model and a very good bioavailability in different species. However, it was shown to induce hyperbilirubinemia in preclinical studies, so it was modified in order to avoid this effect and to improve its pharmacokinetic properties and in vivo potency. In fact, in a preclinical inflammatory pain model, MK 3901 showed and $\mathrm{EC}_{90}=3 \mu \mathrm{M}$ while its improved derivative 21 exerted an $\mathrm{EC}_{90}$ of $0.16 \mu \mathrm{M}$ (Ginnetti et al., 2018). The allosteric potent and selective P2X3R antagonist belonging to arylamide derivatives 3-(5-methylthiazol-2-yl)-5-(((R)-tetrahydrofuran-3-yl)oxy)- $N-((R)$ 1-(2-(trifluoromethyl)pyrimidin-5-yl)ethyl)benzamide (eliapixant also called BAY-1817080, 22; $\mathrm{IC}_{50}=8 \mathrm{nM}$ ), developed by Bayer, showed a dose dependent reduction in cough frequency with low effect on taste perception in a phase IIb clinical trials. Very recently for this compound the company planed Phase II clinical trials for different pathology like diabetic neuropathies and overactive bladder and Phase I clinical trial for endometriosis (NCT04545580, 2020; NCT04614246, 2020).

Also pyrrolinone derivatives have been identified as a novel class of P2X3 receptor antagonists by Shionogi company. Among them, the 5-cyclohexyl-3-hydroxy-1-(4-(isoxazol-4-yl)phenyl)4-(4-methoxybenzoyl)-1,5-dihydro- $2 \mathrm{H}$-pyrrol-2-one resulted a very potent antagonist of P2X3Rs with an $\mathrm{IC}_{50}$ of $0.025 \mu \mathrm{M}$ and a good analgesic efficacy in an acetic acid induced writhing test. Its selectivity was proved respect to 41 receptors and 17 enzymes (Tobinaga et al., 2018).

Among selective P2X3R antagonists there are novel imidazopyridine developed by Biopharmaceutical Company BELLUS Health with the aim to avoid the unpleasant loss of taste which characterized $\mathrm{P} 2 \mathrm{X} 3$ and $\mathrm{P} 2 \mathrm{X} 2 / 3 \mathrm{R}$ s unselective ligand. The most promising seemed to be methyl (S)-3-((2- (2,6difluoro-4-(methylcarbamoyl)phenyl)-7-methylimidazo [1,2-a] pyridin-3-yl)methyl)piperidine-1-carboxylate (BLU-5937, 24; $\mathrm{IC}_{50}$ of $0.025 \mu \mathrm{M}$ and $>24 \mu \mathrm{M}$ at human P2X3 and P2X2/3Rs, respectively) (Pharmacompass, 2018), a compound that demonstrated a good oral availability and pharmacokinetic profile. Differently from gefapixant, BLU-5937 did not affect taste function, even at high doses, in a two bottle taste study (Garceau and Chauret, 2019). The lack of this side effect was 
demonstrated also in a Phase II clinical study, however it didn't reach the primary endpoint since it did not achieve statistical significance, at any dose tested, for the reduction of cough frequency vs. placebo (Evaluate Ltd, 2020). BLU-5937 is currently under clinical evaluation (phase II) for the treatment of chronic pruritus in adult subjects with atopic dermatitis (NCT04693195, 2021).

Another selective P2X3 allosteric blocker, S-600918, developed by Shionogi (structure not reported), is currently being studied in Phase II clinical trials in patients suffering from refractory chronic cough (Dicpinigaitis et al., 2020; Jacobson et al., 2020). This compound showed to induce a reduction of cough frequency vs. placebo with moderate alteration of taste sensitivity in a Phase IIa (JapicCTI-184027, 2019) clinical trial (Niimi et al., 2019). Recruitment is already terminated for the Phase IIb (NCT04110054, 2020) clinical trial, consistent in a randomised, double-blind, placebo-controlled study to assess the onset of efficacy and determine the optimal dose (Ishihara et al., 2020).

\section{DISCUSSION}

The limited distribution of $\mathrm{P} 2 \mathrm{X} 3$ and $\mathrm{P} 2 \mathrm{X} 2 / 3 \mathrm{Rs}$ and their role in mediating painful stimuli in the primary sensory neurons of DRG make these receptors attractive targets in different pathologies. In fact, P2X3 and P2X2/3R antagonists, which inhibit ATP mediating effects on these receptors, represent very interesting tools that could lead to potential analgesic drugs. The advantage of these molecules is their peripheral action, which avoids sedation, gastrointestinal or cardiovascular side effects typical of the current analgesic drugs. In fact, while the availability of selective agonists of these receptors is still a challenge, in recent years many research efforts have led to the discovery of orthosteric and allosteric antagonists belonging to different chemical class. The availability of reliable experimental 3D structures of the P2X3R is a critical factor for the depiction of the mechanism of action of known orthosteric and allosteric ligands and for computational studies aimed at virtually screening or structure-based designing novel potential modulators. Nevertheless, for some compounds endowed with an allosteric mechanism of inhibition and structurally unrelated to the cocrystallized compound gefapixant, the structural determinants at the basis of their interaction with the receptor are still unknown. Molecular modeling studies or further X-Ray crystallography or electron cryomicroscopy experiments could be of help to depict their mechanisms of action and for the design of novel inhibitors.

Compared to marketed orally bioavailable analgesic drugs, the first generation of $\mathrm{P} 2 \mathrm{X} 3$ antagonists preclinically showed a not suitable drug-like profile based on their poor P2X subtype selectivity and/or unfavorable pharmacokinetic/ pharmacodynamic profile (i.e. limited adherence to the Lipinski rules, toxicity, interaction with other drugs) (Gum et al., 2012). These factors compromised their clinical development. An example is given by the first P2X3 antagonist preclinical candidate discovered by Merck, MK-3901 (20,
Figure 1). As already mentioned, in preclinical studies this molecule showed relevant side effects (hyperbilirubinemia), low metabolic stability, and cytochrome P450 inhibition potentially leading to drug-drug interaction (Ginnetti et al., 2018).

More recent P2X3 antagonists were hence developed with improved chemical-physical properties to achieve a drug-like profile combined with high efficacy at the P2X3 receptors. At present, four P2X3 and P2X2/3R allosteric antagonists are under evaluation in different phases of clinical trials for the treatment of patients suffering of overactive bladder, diabetic neuropathies, endometriosis, and refractory chronic cough. For this last pathology, it seems that unselective ligands which bock both the P2X3 and P2X2/3Rs led to a loss of taste as undesirable side effect that seems to be avoided with the use of selective P2X3R antagonists. In fact, the selective allosteric blocker BLU-5937 did not affect taste function, however failed to reach its primary point. Hence, the selective blocker eliapixant or the unselective antagonist gefapixant, which has recently completed two Phase III clinical trials could be the new approved drug for the treatment of refractory chronic cough.

\section{PERSPECTIVES}

Treatment of pain associated to diseases is still a challenge, and a small percentage of sufferers receive adequate prescription for treatment. The restricted localizations of P2X3 and P2X2/3Rs on nociceptive neurons make them an attractive target for the management of inflammatory, neuropathic, and visceral pain states and chronic and refractory chronic cough. The lack of central, gastrointestinal and cardiovascular effects of molecules that block these receptors has greatly stimulated the synthesis of P2X3R antagonists. Most of these molecules are allosteric modulators and some of them are under evaluation in clinical trials. The most relevant side effect of gefapixant is the dysgeusia, which is attenuated or absent in the case of eliapixant and BLU-5937. For the refractory and chronic cough BLU-5937 did not reach its primary end point while gefapixant, although its effect on taste perception, completed phase III clinical trial. Blu is at present evaluated in clinical trial for endometriosis associated pain and chronic pruritus associated atopic dermatitis while eliapixant is under evaluation for endometriosis and chronic cough. We are confident that in the next future these molecules will reach the market as new analgesic and antitussive drugs.

\section{AUTHOR CONTRIBUTIONS}

All authors listed have made a substantial, direct, and intellectual contribution to the work and approved it for publication.

\section{ACKNOWLEDGMENTS}

This work was supported by Fondo di Ricerca di Ateneo (University of Camerino). 


\section{REFERENCES}

Andersson, K.-E. (2016). Potential future pharmacological treatment of bladder dysfunction. Basic Clin. Pharmacol. Toxicol. 119 (Suppl. 3), 75-85. doi:10.1111/ bcpt. 12577

Browne, L. E., Jiang, L.-H., and North, R. A. (2010). New structure enlivens interest in P2X receptors. Trends Pharmacol. Sci. 31 (5), 229-237. doi:10.1016/j.tips. 2010.02.004

Burnstock, G. (2018). The therapeutic potential of purinergic signalling. Biochem. Pharmacol. 151, 157-165. doi:10.1016/j.bcp.2017.07.016

Carter, D. S., Alam, M., Cai, H., Dillon, M. P., Ford, A. P. D. W., Gever, J. R., et al. (2009). Identification and SAR of novel diaminopyrimidines. Part 1: the discovery of RO-4, a dual P2X3/P2X2/3 antagonist for the treatment of pain. Bioorg. Med. Chem. Lett. 19 (6), 1628-1631. doi:10.1016/j.bmcl.2009. 02.003

Dal Ben, D., Buccioni, M., Lambertucci, C., Marucci, G., Spinaci, A., Marchenkova, A., et al. (2019). Investigation on $2^{\prime}, 3^{\prime}$-O-substituted ATP derivatives and analogs as novel P2X3 receptor antagonists. ACS Med. Chem. Lett. 10 (4), 493-498. doi:10.1021/acsmedchemlett.8b00524

Dal Ben, D., Buccioni, M., Lambertucci, C., Marucci, G., Thomas, A., and Volpini, R. (2015). Purinergic P2X receptors: structural models and analysis of ligandtarget interaction. Eur. J. Med. Chem. 89, 561-580. doi:10.1016/j.ejmech.2014. 10.071

Dal Ben, D., Marchenkova, A., Thomas, A., Lambertucci, C., Spinaci, A., Marucci, G., et al. (2017). 2',3'-O-Substituted ATP derivatives as potent antagonists of purinergic P2X3 receptors and potential analgesic agents. Purinergic Signal. 13 (1), 61-74. doi:10.1007/s11302-016-9539-y

De Logu, F., and Geppetti, P. (2019). ion channel pharmacology for pain modulation. Handb. Exp. Pharmacol. 260, 261-289. doi:10.1007/164-2019-336

Dicpinigaitis, P. V., McGarvey, L. P., and Canning, B. J. (2020). P2X3-Receptor antagonists as potential antitussives: summary of current clinical trials in chronic cough. Lung 198 (4), 609-616. doi:10.1007/s00408-020-00377-8

Dillon, M. P., Hawley, R. C., Chen, L., Feng, L., and Minmin, Y. (2015). Tetrazolesubstituted arylamides as P2X3 and P2X2/3 antagonists. US. $0191487 \mathrm{~A}$.

Evaluate Ltd (2020). Bellus chokes in chronic cough https://www.evaluate.com/ vantage/articles/news/trial-results/bellus-chokes-chronic-cough (Accessed January 13, 2021).

Ford, A. P. D. W., and Cockayne, D. A. (2011). ATP and P2X purinoceptors in urinary tract disorders. Handb. Exp. Pharmacol., 485-526. doi:10.1007/978-3642-16499-6_22

Ford, A. P. D. W., Gever, J. R., Nunn, P. A., Zhong, Y., Cefalu, J. S., Dillon, M. P., et al. (2006). Purinoceptors as therapeutic targets for lower urinary tract dysfunction. Br. J. Pharmacol. 147 (Suppl. 2), S132-S143. doi:10.1038/sj.bjp.0706637

Ford, A. P. (2012). In pursuit of P2X3 antagonists: novel therapeutics for chronic pain and afferent sensitization. Purinergic Signal. 8 (Suppl 1), 3-26. doi:10. 1007/s11302-011-9271-6

Ford, A. P., and Undem, B. J. (2013). The therapeutic promise of ATP antagonism at $\mathrm{P} 2 \mathrm{X} 3$ receptors in respiratory and urological disorders. Front. Cel. Neurosci. 7, 267. doi:10.3389/fncel.2013.00267

Fredholm, B. B., IJzerman, A. P., Jacobson, K. A., Linden, J., and Müller, C. E. (2011). International union of basic and clinical pharmacology. LXXXI. Nomenclature and classification of adenosine receptors-an update. Pharmacol. Rev. 63 (1), 1-34. doi:10.1124/pr.110.003285

Garceau, D., and Chauret, N. (2019). BLU-5937: a selective P2X3 antagonist with potent anti-tussive effect and no taste alteration. Pulm. Pharmacol. Ther. 56, 56-62. doi:10.1016/j.pupt.2019.03.007

Gever, J. R., Soto, R., Henningsen, R. A., Martin, R. S., Hackos, D. H., Panicker, S., et al. (2010). AF-353, a novel, potent and orally bioavailable P2X3/P2X2/3 receptor antagonist. Br. J. Pharmacol. 160 (6), 1387-1398. doi:10.1111/j.14765381.2010.00796.x

Giniatullin, R., and Nistri, A. (2013). Desensitization properties of P2X3 receptors shaping pain signaling. Front. Cel. Neurosci. 7, 245. doi:10.3389/fncel.2013. 00245

Ginnetti, A. T., Paone, D. V., Stauffer, S. R., Potteiger, C. M., Shaw, A. W., Deng, J., et al. (2018). Identification of second-generation P2X3 antagonists for treatment of pain. Bioorg. Med. Chem. Lett. 28 (8), 1392-1396. doi:10.1016/ j.bmcl.2018.02.039
Grimes, L., and Young, M. (2015). Purinergic P2X receptors: structural and functional features depicted by X-ray and molecular modelling studies. Cmc 22 (7), 783-798. doi:10.2174/0929867321999141212131457

Gum, R. J., Wakefield, B., and Jarvis, M. F. (2012). P2X receptor antagonists for pain management: examination of binding and physicochemical properties. Purinergic Signal. 8 (Suppl 1), 41-56. doi:10.1007/s11302-011-9272-5

Honore, P., Mikusa, J., Bianchi, B., McDonald, H., Cartmell, J., Faltynek, C., et al. (2002). TNP-ATP, a potent P2X3 receptor antagonist, blocks acetic acidinduced abdominal constriction in mice: comparison with reference analgesics. Pain 96 (1-2), 99-105. doi:10.1016/s0304-3959(01)00434-1

Illes, P., Müller, C. E., Jacobson, K. A., Grutter, T., Nicke, A., Fountain, S. J., et al. (2020). Update of P2X receptor properties and their pharmacology: IUPHAR Review 30. Br. J. Pharmacol. 178 (3), 489-514. doi:10.1111/bph.15299

Ishchenko, Y., Shakirzyanova, A., Giniatullina, R., Skorinkin, A., Bart, G., Turhanen, P., et al. (2017). SAgonists and antagonists for P2 receptors. J. Pharmacol. Exp. Ther. 361 (3), 472-481. doi:10.1124/jpet.116.238840

Ishihara, H., Hida, H., Machida, M., Tsuda, Y., and Miyazaki, S. (2020). Design of phase $2 \mathrm{~b}$ randomised controlled trial of S-600918, P2X3 receptor antagonist for refractory chronic cough. Eur. Respir. J. 56, 2271. doi:10.1183/13993003. congress-2020.2271

Jacobson, K. A., Costanzi, S., Joshi, B. V., Besada, P., Shin, D. H., Ko, H., et al. (2006). Agonists and antagonists for P2 receptors. Novartis Found. Symp. 276, 58-81. doi:10.1002/9780470032244.ch6

Jacobson, K. A., IJzerman, A. P., and Müller, C. E. (2020). Medicinal chemistry of P2 and adenosine receptors: common scaffolds adapted for multiple targets. Biochem. Pharmacol. 114311. doi:10.1016/j.bcp.2020.114311

Jacobson, K. A., Jarvis, M. F., and Williams, M. (2002). Purine and pyrimidine (P2) receptors as drug targets. J. Med. Chem. 45, 4057-4093. doi:10.1021/jm020046y

JapicCTI-184027 (2019). A Phase 2, randomized, double-blind, cross-over study of S-600918 in subjects with refractory/unexplained chronic cough (ClinicalTrials.jp Identifier) https://www.clinicaltrials.jp/cti-user/trial/ ShowDirect.jsp?japicId=JapicCTI-184027 (Accessed February 15, 2021).

Jarvis, M. F., Burgard, E. C., McGaraughty, S., Honore, P., Lynch, K., Brennan, T. J., et al. (2002). A-317491, a novel potent and selective non-nucleotide antagonist of $\mathrm{P} 2 \mathrm{X} 3$ and $\mathrm{P} 2 \mathrm{X} 2 / 3$ receptors, reduces chronic inflammatory and neuropathic pain in the rat. Proc. Natl. Acad. Sci. 99 (26), 17179-17184. doi:10.1073/pnas. 252537299

Jarvis, M. F. (2003). Contributions of P2X3 homomeric and heteromeric channels to acute and chronic pain. Expert Opin. Ther. Targets 7 (4), 513-522. doi:10. $1517 / 14728222.7 .4 .513$

Jarvis, M. F. (2020). Geoffery Burnstock's influence on the evolution of P2X3 receptor pharmacology. Purinergic Signal. 17 (1), 33-39. doi:10.1007/s11302-020-09744-9

Jarvis, M. F., and Khakh, B. S. (2009). ATP-gated P2X cation-channels Neuropharmacology 56, 208-215. doi:10.1016/j.neuropharm.2008.06.067

Kakimoto, S., Nagakura, Y., Tamura, S., Watabiki, T., Shibasaki, K., Tanaka, S., et al. (2008). Minodronic acid, a third-generation bisphosphonate, antagonizes purinergic $\mathrm{P} 2 \mathrm{X} 2 / 3$ receptor function and exerts an analgesic effect in pain models. Eur. J. Pharmacol. 589 (1-3), 98-101. doi:10.1016/j.ejphar.2008.05.011

Kennedy, C. (2005). P2X receptors: targets for novel analgesics? Neuroscientist 11 (4), 345-356. doi:10.1177/1073858404274063

Lambertucci, C., Ben, D., Buccioni, M., Marucci, G., Thomas, A., and Volpini, R. (2015). Medicinal chemistry of P2X receptors: agonists and orthosteric antagonists. Cmc 22 (7), 915-928. doi:10.2174/0929867321666141215093513

Lambertucci, C., Sundukova, M., Kachare, D. D., Panmand, D. S., Dal Ben, D., Buccioni, M., et al. (2013). Evaluation of adenine as scaffold for the development of novel P2X3 receptor antagonists. Eur. J. Med. Chem. 65, 41-50. doi:10.1016/j.ejmech.2013.04.037

Lewis, C. J., Surprenant, A., and Evans, R. J. (1998). 2',3'-O-(2,4,6- trinitrophenyl) adenosine $5^{\prime}$-triphosphate (TNP-ATP)-a nanomolar affinity antagonist at rat mesenteric artery P2X receptor ion channels. Br. J. Pharmacol. 124, 1463-1466. doi:10.1038/sj.bjp.0702001

Lewis, C., Neidhart, S., Holy, C., North, R. A., Buell, G., and Surprenant, A. (1995). Coexpression of P2X2 and P2X3 receptor subunits can account for ATP-gated currents in sensory neurons. Nature 377, 432-435. doi:10.1038/377432a0

Li, M., Wang, Y., Banerjee, R., Marinelli, F., Silberberg, S., Faraldo-Gómez, J. D., et al. (2019). Molecular mechanisms of human P2X3 receptor channel activation and modulation by divalent cation bound ATP. Elife 8, e47060. doi:10.7554/eLife.47060 
Mansoor, S. E., Lü, W., Oosterheert, W., Shekhar, M., Tajkhorshid, E., and Gouaux, E. (2016). X-ray structures define human P2X3 receptor gating cycle and antagonist action. Nature 538 (7623), 66-71. doi:10.1038/nature19367

Marucci, G., Dal Ben, D., Buccioni, M., Martí Navia, A., Spinaci, A., Volpini, R., et al. (2019). Update on novel purinergic $\mathrm{P} 2 \mathrm{X} 3$ and $\mathrm{P} 2 \mathrm{X} 2 / 3$ receptor antagonists and their potential therapeutic applications. Expert Opin. Ther. Patents 29 (12), 943-963. doi:10.1080/13543776.2019.1693542

McDonald, H. A., Chu, K. L., Bianchi, B. R., McKenna, D. G., Briggs, C. A., Burgard, E. C., et al. (2002). Potent desensitization of human P2X3 receptors by diadenosine polyphosphates. Eur. J. Pharmacol. 435 (2-3), 135-142. doi:10. 1016/s0014-2999(01)01568-0

Merck News release (2020). Merck's gefapixant (45 mg twice daily) significantly decreased cough frequency compared to placebo at week 12 and 24 in patients with refractory or unexplained chronic cough https://www.merck.com/news/ mercks-gefapixant-45-mg-twice-daily-significantly-decreased-cough-frequencycompared-to-placebo-at-week-12-and-24-in-patients-with-refractory-orunexplained-chronic-cough (Accessed February 16, 2021).

Muccino, D. R., Morice, A. H., Birring, S. S., Dicpinigaitis, P. V., Pavord, I. D., Assaid, C., et al. (2020). Design and rationale of two phase 3 randomised controlled trials (COUGH-1 and COUGH-2) of gefapixant, a P2X3 receptor antagonist, in refractory or unexplained chronic cough. ERJ Open Res. 6 (4), 00284-2020. doi:10.1183/23120541.00284-2020

NCT03449134 (2020). Phase 3 study of gefapixant (MK-7264) in adult participants with chronic cough (MK-7264-027) (ClinicalTrials.gov identifier) https:// clinicaltrials.gov/ct2/show/NCT03449134 (Accessed January 11, 2021).

NCT03449147 (2020). A Study of Gefapixant (MK-7264) in Adult Participants With Chronic Cough (MK-7264-030) (ClinicalTrials.gov identifier) https:// clinicaltrials.gov/ct2/show/NCT03449147 (Accessed January 08, 2021).

NCT04110054 (2020). Evaluation of S-600918 in adults with refractory chronic cough (ClinicalTrials.gov identifier) https://clinicaltrials.gov/ct2/show/ NCT04110054 (Accessed February 10, 2021).

NCT04545580 (2020). Clinical study to evaluate the treatment effect and safety of $B A Y 1817080$ in patients with overactive bladder (OAB) (OVADER) (ClinicalTrials.gov identifier) https://clinicaltrials.gov/ct2/show/ NCT04545580 (Accessed January 12, 2021).

NCT04614246 (2020). Study to gather information how well three different doses of BAY1817080 given twice daily over 12 Weeks work in comparison to an inactive pill (placebo) and elagolix in women suffering from pain related to a condition where the tissue that usually grows inside the womb grows outside of the womb (SCHUMANN) (ClinicalTrials.gov identifier) https://clinicaltrials.gov/ct2/ show/NCT04614246 (Accessed January 13, 2021).

NCT04693195 (2021). A multiple-dose study of BLU-5937 in chronic pruritus associated with atopic dermatitis (blueprint) (ClinicalTrials.gov identifier) https://clinicaltrials.gov/ct2/show/NCT04693195 (Accessed January 05, 2021).

Niimi, A., Ishihara, H., Hida, H., and Miyazaki, S. (2019). Late Breaking Abstract phase 2a randomised, double-blind, placebo-controlled, crossover study of a novel P2X3 receptor antagonist S-600918 in patients with refractory chronic cough. Eur. Respir. J. 54, RCT452. doi:10.1183/13993003.congress-2019.RCT452
North, R. A., and Surprenant, A. (2000). Pharmacology of cloned P2X receptors Annu. Rev. Pharmacol. Toxicol. 40 (1), 563-580. doi:10.1146/annurev. pharmtox.40.1.563

Ohishi, T., and Matsuyama, Y. (2018). Minodronate for the treatment of osteoporosis. Tcrm 14, 729-739. doi:10.2147/TCRM.S149236

Pelleg, A., Xu, F., Zhuang, J., Undem, B., and Burnstock, G. (2019). DT-0111: a novel drug-candidate for the treatment of COPD and chronic cough. Ther. Adv. Respir. Dis. 13, 175346661987796. doi:10.1177/1753466619877960

Pharmacompass (2018). Pharmacompass entry: BLU-5937 https://www. pharmacompass.com/active-pharmaceutical-ingredients/blu-5937 (Accessed January 04, 2021).

Serrano, A., Mo, G., Grant, R., Pare, M., O’Donnell, D., Yu, X. H., et al. (2012). Differential expression and pharmacology of native $\mathrm{P} 2 \mathrm{X}$ receptors in rat and primate sensory neurons. J. Neurosci. 32 (34), 11890-11896. doi:10.1523/ JNEUROSCI.0698-12.2012

Tobinaga, H., Kameyama, T., Oohara, M., Kobayashi, N., Ohdan, M., Ishizuka, N., et al. (2018). Pyrrolinone derivatives as a new class of P2X3 receptor antagonists. Part 1: initial structure-activity relationship studies of a hit from a high throughput screening. Bioorg. Med. Chem. Lett. 28 (13), 2338-2342. doi:10.1016/j.bmcl.2017.04.060

Viatchenko-Karpinski, V., Novosolova, N., Ishchenko, Y., Azhar, M. A., Wright, M., Tsintsadze, V., et al. (2016). Stable, synthetic analogs of diadenosine tetraphosphate inhibit rat and human P2X3 receptors and inflammatory pain. Mol. Pain 12, 174480691663770. doi:10.1177/1744806916637704

Volpini, R., Mishra, R. C., Kachare, D. D., Ben, D. D., Lambertucci, C., Antonini, I., et al. (2009). Adenine-based acyclic nucleotides as novel P2X3Receptor ligands. J. Med. Chem. 52 (15), 4596-4603. doi:10.1021/jm900131v

Wang, J., Wang, Y., Cui, W.-W., Huang, Y., Yang, Y., Liu, Y., et al. (2018). Druggable negative allosteric site of $\mathrm{P} 2 \mathrm{X} 3$ receptors. Proc. Natl. Acad. Sci. USA 115 (19), 4939-4944. doi:10.1073/pnas.1800907115

Weigand, L. A., Ford, A. P., and Undem, B. J. (2012). A role for ATP in bronchoconstriction-induced activation of Guinea pig vagal intrapulmonary C-fibres. J. Physiol. 590 (16), 4109-4120. doi:10.1113/jphysiol.2012.233460

Wildman, S. S., Brown, S. G., King, B. F., and Burnstock, G. (1999). Selectivity of diadenosine polyphosphates for rat P2X receptor subunits. Eur. J. Pharmacol. 367 (1), 119-123. doi:10.1016/s0014-2999(98)00976-5

Conflict of Interest: The authors declare that the research was conducted in the absence of any commercial or financial relationships that could be construed as a potential conflict of interest.

Copyright (c) 2021 Spinaci, Buccioni, Dal Ben, Marucci, Volpini and Lambertucci. This is an open-access article distributed under the terms of the Creative Commons Attribution License (CC BY). The use, distribution or reproduction in other forums is permitted, provided the original author(s) and the copyright owner(s) are credited and that the original publication in this journal is cited, in accordance with accepted academic practice. No use, distribution or reproduction is permitted which does not comply with these terms. 